\title{
Thermal Analysis of Blue Laser Diode for Solid State Lighting Application
}

\author{
Ada Czesnakowska1* ${ }^{*}$, Gerald Ledru1, Benoit Glorieux², Georges Zissis ${ }^{1}$ \\ ${ }^{1}$ Laplace Laboratory, University of Toulouse III, Toulouse, France \\ ${ }^{2}$ Institut de Chimie de la Matière Condensée de Bordeaux, CNRS, Pessac, France \\ Email: *czesnakowska@laplace.univ-tlse.fr, ledru@laplace.univ-tlse.fr
}

How to cite this paper: Czesnakowska, A. Ledru, G., Glorieux, B. and Zissis, G. (2018) Thermal Analysis of Blue Laser Diode for Solid State Lighting Application. Optics and Photonics Journal, 8, 40-49. https://doi.org/10.4236/opj.2018.83005

Received: February 17, 2018

Accepted: March 26, 2018

Published: March 29, 2018

Copyright (c) 2018 by authors and Scientific Research Publishing Inc. This work is licensed under the Creative Commons Attribution International License (CC BY 4.0).

http://creativecommons.org/licenses/by/4.0/

(c) (i) Open Access

\begin{abstract}
Solid-state light sources based on laser diode are becoming great alternative for LEDs. Improvement of the thermal characteristics of InGaN LD is very important for realizing reliable devices. In this investigation the influence of the temperature of diode on light parameters was studied. White light was obtained by coupling blue light of diode with yellow phosphors: YAG:Ce ${ }^{3+}$ and GYAG:Ce ${ }^{3+}$ with nitride. For three values of the temperature of LD's stem, regulated by Peltier module, CCT, CRI and chromaticity coordinates were measured by spectroradiometer. The importance of emission characteristics of materials was shown. Subsequently, the influence of temperature on laser diode intensity was investigated for 120 hours. This experiment was repeated for different levels of current and temperature. Finally, the steady state of thermal finite element analysis was performed to reveal the distribution of the temperature. The analysis showed the importance of heat sink and also temperature control.
\end{abstract}

\section{Keywords}

Laser Diode, Solid State Lighting, YAG, FEM, Thermal Analysis

\section{Introduction}

In past few years InGaN-based semiconductors have attracted much attention for application in solid-state light sources. Recently, their usage is constantly increasing in worldwide market. High-brightness white LEDs have been used due to their size, long life and energy saving. Also, white light can be generated from LEDs in three major ways: mixing blue, red and green diode [1] [2], coupling UV diode with RGB phosphor [3] or coupling blue diode with yellow phosphor [4]. The last method is the most convenient and efficient to generate white light 
and it has been commonly adapted in white LEDs.

Unfortunately, LEDs used in these devices suffer a loss in external quantum efficiency as operating current increases, known as a droop. This may lead to shift in a peak emission wavelength and broadening of the emission spectrum. Variations of spectrum of LED and phosphor change the ratio of the light emitted by each component, resulting in shift in the color coordinates of the emitted light and decrease in overall device efficiency [5].

An alternative to light-emitting diodes for obtaining white light can be blue laser diode (LD). In contrast to LEDs, they do not exhibit efficiency loss. The output power and external quantum efficiency of laser diode increase linearly with current and maintains color stability of laser emission. Additionally, laser-based devices can be operated in reflection mode, allowing for the phosphor to be placed on a reflection substrate that may also act as a heat sink to effectively dissipate heat away from the phosphor.

Common problem of SSL based on semiconductors is their heating [6] [7] [8], when the current increases. It doesn't concern only lighting domain, but also data storage or communication, when coupling of laser and phosphor is used [9] [10]. To avoid the diode degradation caused by heat and to assure long-life, the cooling module is required. In our investigation we verified the influence of the temperature augmentation of the laser diode on parameters of the light generated by coupling the laser diode and phosphor. We also studied the distribution of the temperature in the laser diode by using FEM (Finite Element Method) analysis.

\section{Temperature Augmentation}

\subsection{Experimental Setup}

The white light was generated by coupling blue laser diode and two different types of materials. For the production of the materials a two-part high optical transparency silicon was used as a matrix. Silicon was filled in with different powders to obtain two types of material: YAG:Ce ${ }^{3+}$ (NYAG4354 Intematix) powder and mixture of green GYAG:Ce ${ }^{3+}$ (GYAG3856-01-13 Intematix) and nitride in ratio 4.5:1. Phosphors were incorporated into silicon plate of diameter $10 \mathrm{~mm}$ and thickness of $1 \mathrm{~mm}$. The materials were prepared in ICMCB laboratory in Bordeaux, France. As an excitation source the InGaN blue laser diode OSRAM TB450B $450 \mathrm{~nm}$ was used. Laser was thermally coupled with a Peltier module, which was connected to PID temperature controller TEC 2000 from Thor Labs. A sensor AD590 was placed under a stem of the laser to measure the temperature. The measurements were performed in an optical sphere (Figure 1), where as an output the spectroradiometer SpecBos 1201 was put. In front of spectrometer we placed a phosphor plate. This let us to keep a phosphor in a distance from a diode, to avoid heating of material. The diode with its module was put as an input of the sphere. The spectrometer allowed us to measure spectrum of light, CRI (Color Rendering Index), CCT (Correlated Color Temperature) 


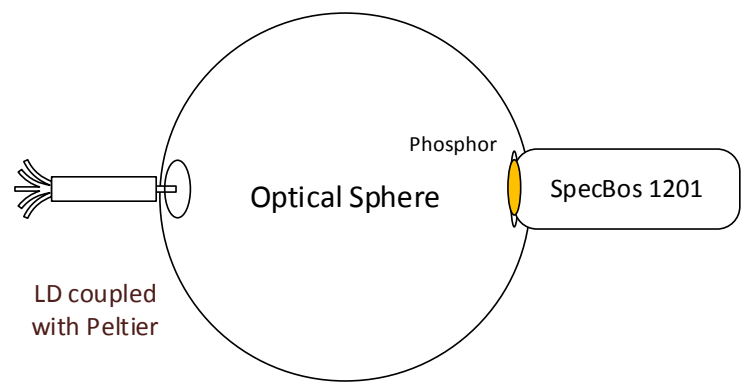

Figure 1. Measurement Setup. Blue laser diode in Peltier module as an input, produces the light that passes by phosphor. The plate of phoshor is placed as an output of sphere, just in front of spectro-radiometer, to avoid heating from the laser itself.

and chromaticity coordinates. The temperature of $\mathrm{LD}$ was fixed at three different levels: first at $25^{\circ} \mathrm{C}$, then at $45^{\circ} \mathrm{C}$ and finally $55^{\circ} \mathrm{C}$. For each, we measured firstly the spectrum of the LD itself, and then the spectrum of laser's light converted by the phosphor and its parameters.

\subsection{Results}

Below, on Figure 2, the spectrum of blue LD for different diode's temperatures was presented. When the temperature increases, the shift into higher wavelengths can be observed. Moreover, when reaching the $55^{\circ} \mathrm{C}$, the intensity of the light decreases about $30 \%$. This shift obviously has influence on the emission of the material (Figure 3 and Figure 4). The graphs were normalized to peak of laser emission in order to present the amount of converted light by the phosphor. On Figure 3 the intensity of converted light increase proportionally to the temperature, but this augmentation is not significant. In contrary, on Figure 4, it can be found that converted light decreased by $50 \%$, only after changing the temperature by $15^{\circ}$.

This variation is connected to the characteristics of light emission by materials. The optimal emission for YAG can be found at level of $442 \mathrm{~nm}$ (Figure 5) and for GYAG: $437 \mathrm{~nm}$ (Figure 6). GYAG was mixed with nitride, so its emission needs also be taken into consideration (Figure 7).

If the emission of converted light drops, at the output of our light source we find more blue light. It is caused by the drop of the efficiency of the phosphor emission. In emission characteristics we are not anymore at the maximum, which means that more blue light passes through plate. Rise of blue color can cause perturbation of human circadian rhythm [11], which can be dangerous to health.

The color rendering index (CRI) of light source defines the ability of the source to render the true colors of an object [12]. The maximum value is 100 , which implies the best possible color rendition. The down-conversion method of obtaining white light has an advantage of a high CRI due to the broad emission spectrum of a phosphor [8]. Based on the results presented in Table 1 we can say that changings in emission spectrum do not influence on CRI parameter. 


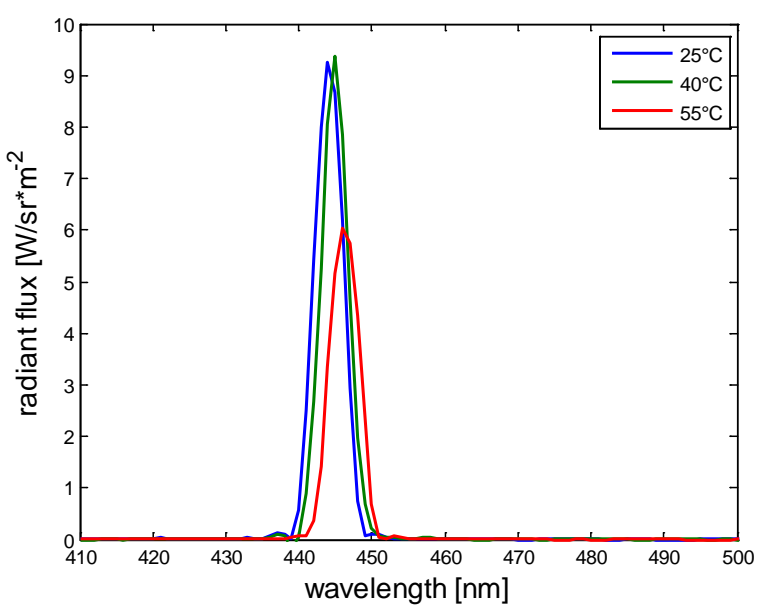

Figure 2. Spectrum of blue laser diode for different temperature.

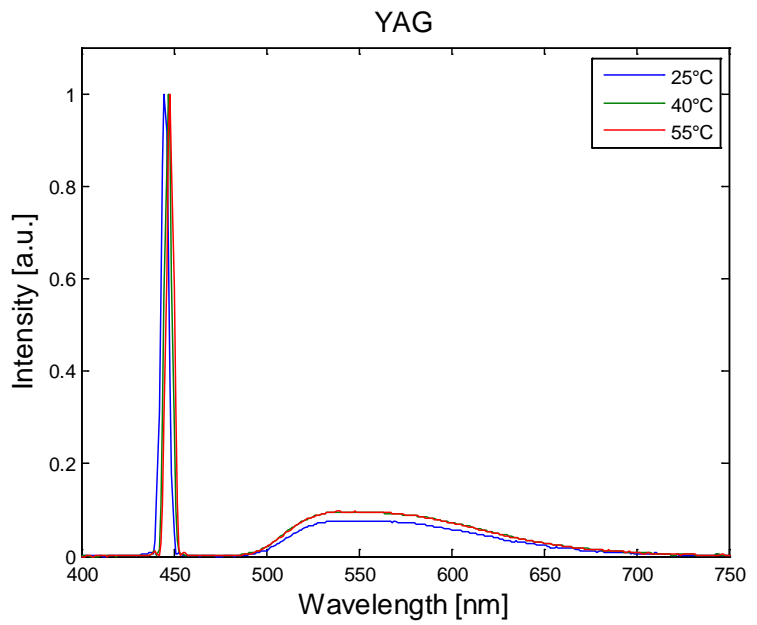

Figure 3. Spectrum of converted light by YAG for different temperature.

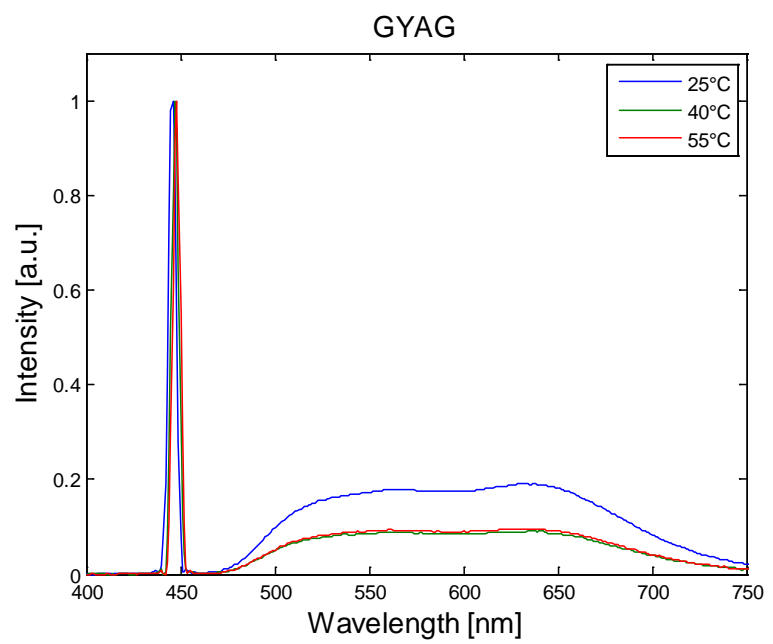

Figure 4. Spectrum of converted light by GYAG for different temperature. 


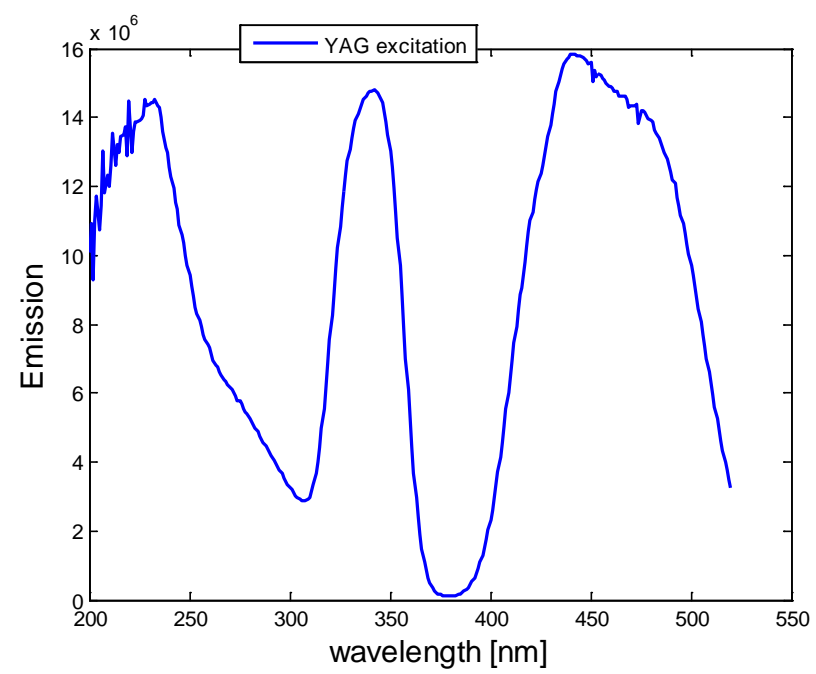

Figure 5. Emission of YAG:Ce ${ }^{3+}$.

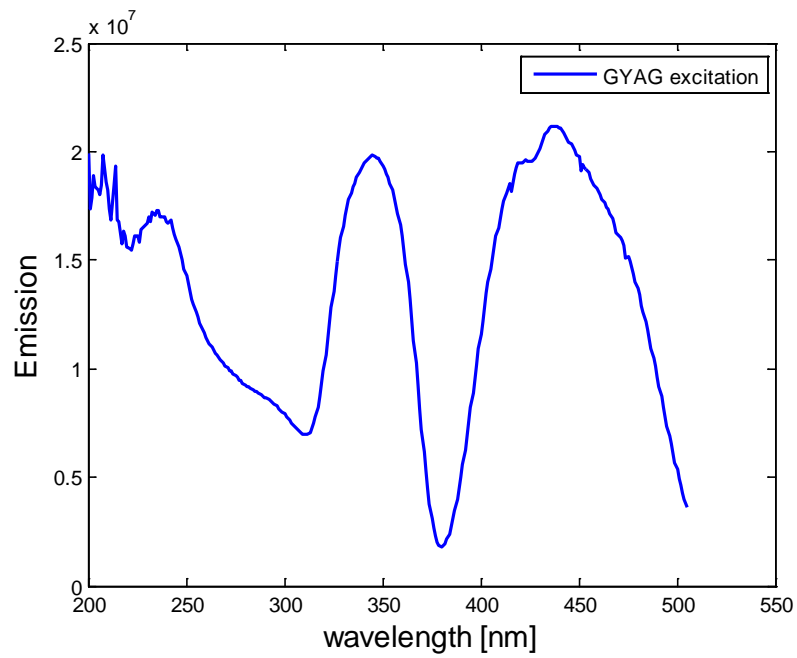

Figure 6. Emission of GYAG:Ce ${ }^{3+}$.

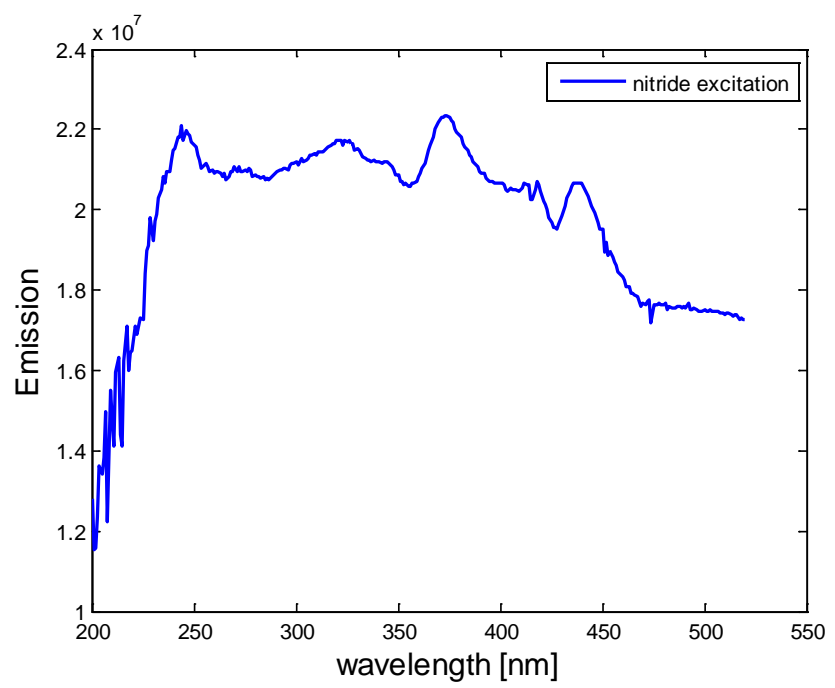

Figure 7. Emission of nitride. 
Table 1. Color rendering index.

\begin{tabular}{ccc}
\hline Temperature & YAG: $\mathrm{Ce}^{3+}$ & GYAG: $\mathrm{Ce}^{3+}$ with nitride \\
\hline $25^{\circ} \mathrm{C}$ & 65 & 85 \\
$40^{\circ} \mathrm{C}$ & 65 & 84 \\
$55^{\circ} \mathrm{C}$ & 65 & 84
\end{tabular}

Since the efficiency of converted material changed, CCT and chromaticity coordinates should be affected. In Table 2 we present CCT values obtained and measured by SpecBos spectrometer. On Figure 8 the chromaticity coordinates were presented.

Emission by YAG:Ce ${ }^{3+}$ does not change radically. The small shift into green can be noticed. In contrary, the shift into blue is clearly seen on the figure above for GYAG: $\mathrm{Ce}^{3+}$, which is caused by drop of the emission efficiency.

Variation of the diode temperature not only has an influence on light parameters. Operating on high value of temperature can lead to reduction of life duration of semiconductor. To obtain a long lifetime of a laser, appropriate cooling is required. The important factor to prolong diode operation is junction temperature not exceeding $100^{\circ} \mathrm{C}$. In our study, we investigated the temperature distribution of the operating diode by performing Finite Element Method Analysis.

\section{FEM Analysis}

The Laplace heat equation was numerically solved for this thermal analysis [13]. For steady state approach the solution is independent from density and specific heat. All the external surfaces were in heat convection with the air. The ambient temperature was fixed at $25^{\circ} \mathrm{C}$. In semiconductor the heat generation is located in active layer and p-cladding layer. In our simulation we defined active layer and p-cladding layer as "heat generators". The value of heat generation was calculated based on lumped element model:

$$
\begin{gathered}
Q_{c}=I V \\
I=W L J \\
V=\frac{h_{l} J}{\sigma}
\end{gathered}
$$

where $Q_{c}$ is the heat generation, $V$ the voltage drop, $J$ the density of current, $W$ the stripe width, $L$ the cavity length, $h$ the high of the layer, $\sigma$ the electrical conductivity.

Below, the model designed in FEM software is presented (Figure 9). Chip is mounted on AlNsubmount in a TO 56 package. Submount is attached to heat sink.

Based on thermal conductivity of each material [6], the distribution of the temperature was simulated. The conditions of simulation on Figure 10 represent case without any radiator or cooling module of a laser diode.

The maximum temperature reached on the chip and substrate was $190.15^{\circ} \mathrm{C}$, which exceed the recommended operating junction temperature almost twice. 
Table 2. Correlated color temperature.

\begin{tabular}{ccc}
\hline Temperature & YAG: $\mathrm{Ce}^{3+}$ & GYAG:Ce $^{3+}$ with nitride \\
\hline $25^{\circ} \mathrm{C}$ & 6653 & 3927 \\
$40^{\circ} \mathrm{C}$ & 6354 & 4974 \\
$55^{\circ} \mathrm{C}$ & 6254 & 4974 \\
\hline
\end{tabular}

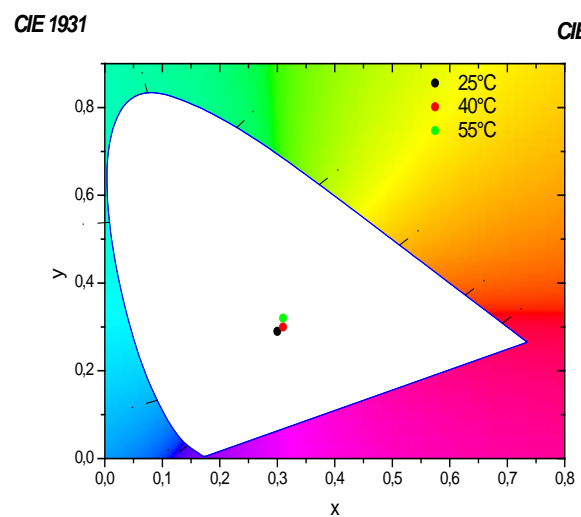

CIE 1931

Figure 8. Chromaticity coordinates changing for YAG:Ce ${ }^{3+}$ and GYAG: $\mathrm{Ce}^{3+}$ with nitride.

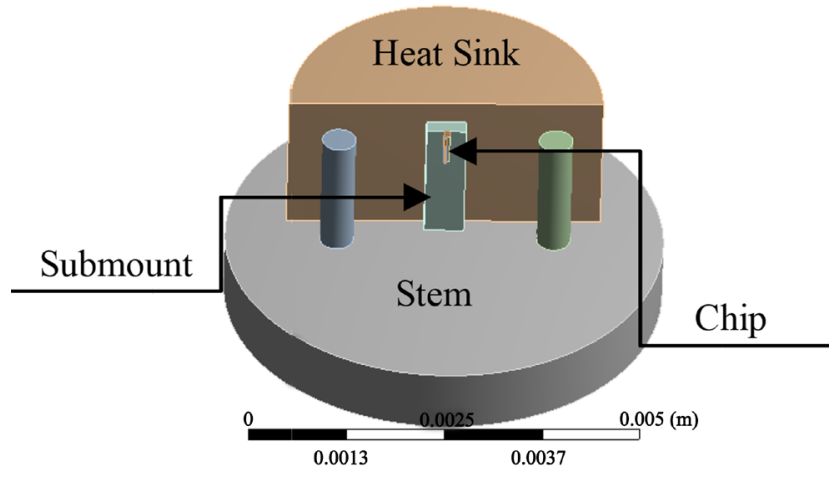

Figure 9. Model of a laser diode for simulation purposes.

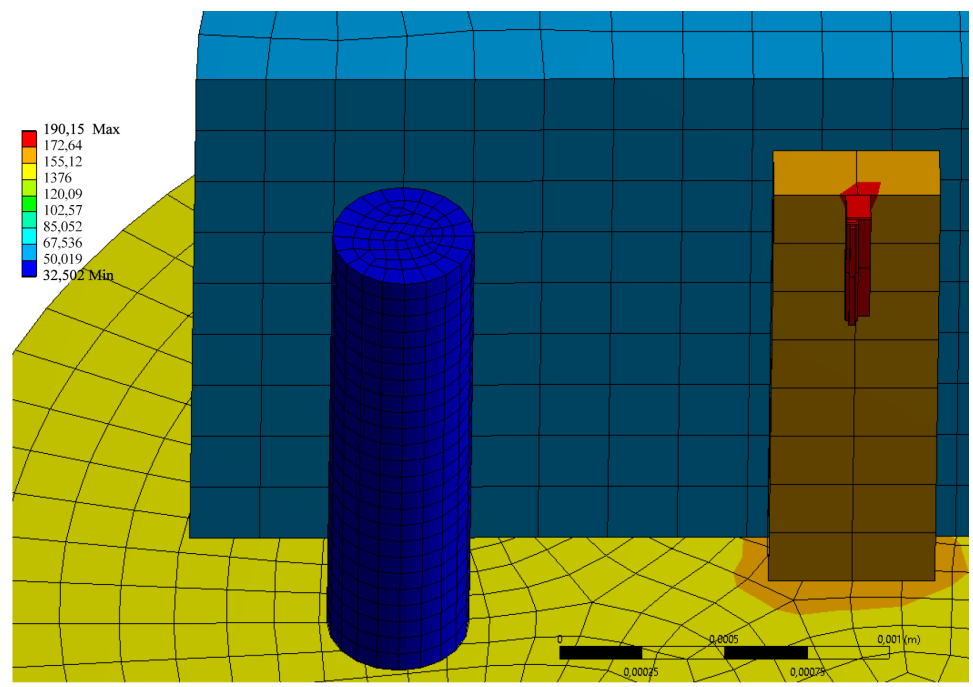

Figure 10. Distribution of temperature without external cooling module. 
The heat dissipation for a packaged semiconductor is mainly by conduction. From the heat source, through the substrate, finally to heat sink. Heat can be also removed by convection via a gas, as in surrounding air. Although effective heat transfers from source to ambient is an aim, it is more usual to define heat dissipation by considering inverse of the heat flow-thermal resistance between these two points. It is calculated by equation:

$$
R_{\text {th }}=\frac{\Delta T}{Q_{\text {total }}}
$$

where $\Delta T$ is a difference of the temperature between junction and ambient, $Q_{\text {total }}$ is a total heat generation. Calculated $R_{t h}$ was $46 \mathrm{~K} / \mathrm{W}$. However, the thermal resistance value calculated by this equation is a lumped value and will differ from depending on the special distribution of the heat source. Subsequently, we simulated the conditions, where we fixed our temperature of stem on $25^{\circ} \mathrm{C}$, and then $55^{\circ} \mathrm{C}$ respectively, which represents thermal coupling with Peltier module.

Figure 11 and Figure 12 show, that fixing proper temperature on stem, by using cooling module and temperature controller let us not exceed the maximum of junction temperature equal to $100^{\circ} \mathrm{C}$. Comparing results of simulation without temperature control, to the one, where the control was used, it is noticed that stem reaches almost $140^{\circ} \mathrm{C}$. Considering, that heat flow is directed toward the heat sink, the temperature of the stem should be lower. The result suggests that the parameters of stem material need to be optimized.

The thermal resistance, while connected thermally to the copper plate, results in the lower value of $15.5 \mathrm{~K} / \mathrm{W}$ (Equation (5)).Studies show [14] [15], that decreasing the connected area at the bottom of the stem will increase in thermal resistance. Also, connecting a diode from lateral surface is enough to keep thermal resistance low. No connection, or connections only to pins lead to even three times increase of this value $(46 \mathrm{~K} / \mathrm{W})$.

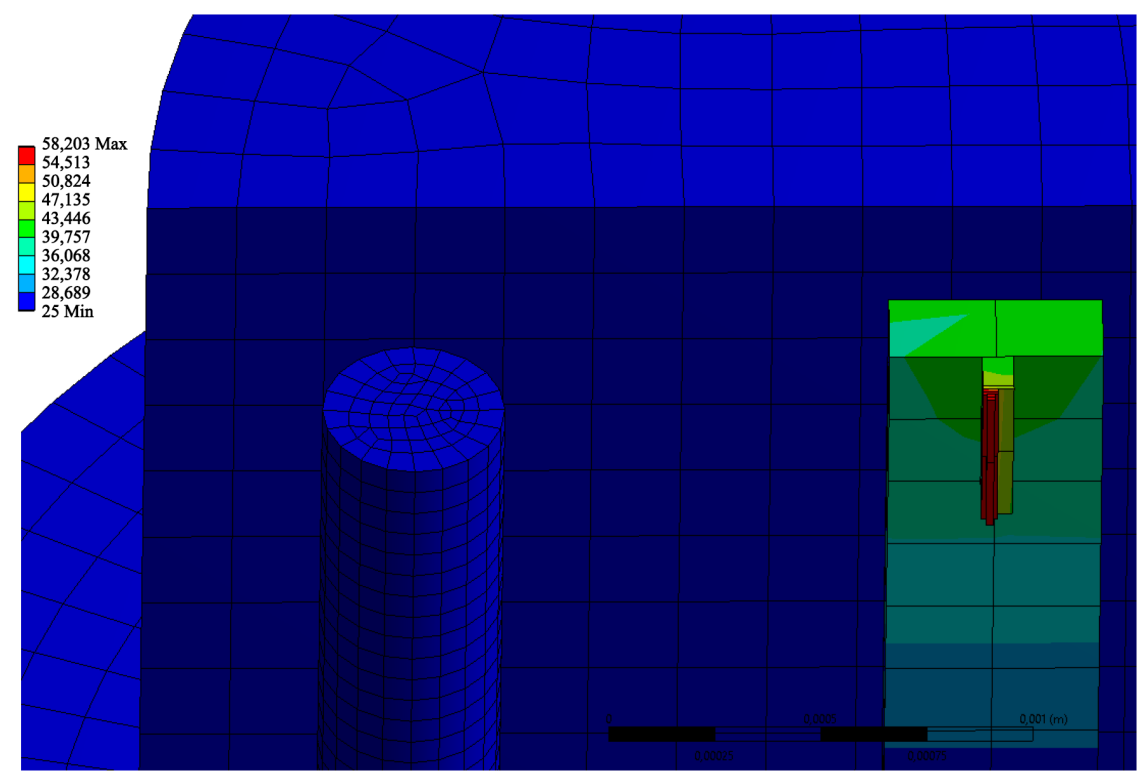

Figure 11. Distribution of temperature for fixed $25^{\circ} \mathrm{C}$ on stem. 


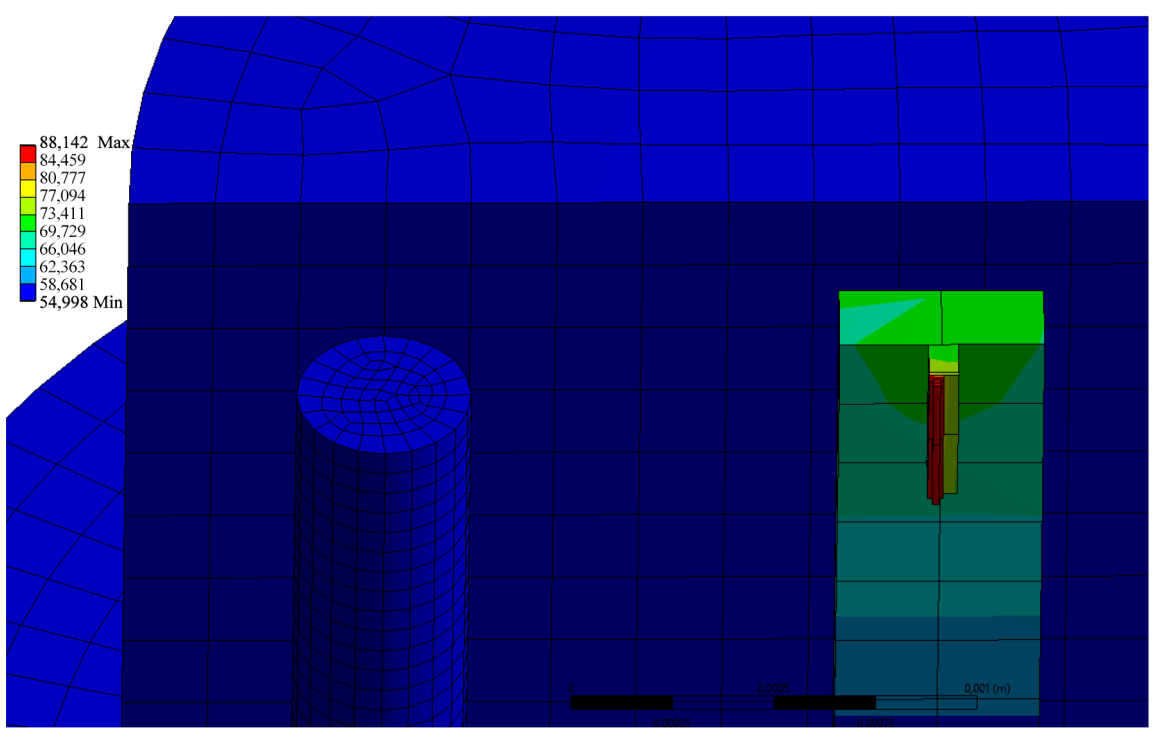

Figure 12. Distribution of temperature for fixed $55^{\circ} \mathrm{C}$ on stem.

\section{Conclusions}

The dependency of the temperature on the emission of a laser, which results in variation of light parameters, when used as excitation source in down converted phosphor method was investigated.

To study the influence of this changing on white light parameters, the phosphors absorption and emission characteristics were performed. Investigation shows that shift of wavelength, which is a result of temperature rise has an influence on light parameter. In case of our blue laser diode coupled with YAG:Ce ${ }^{3+}$, it came out that more optimal results, closer to "daylight" parameters, are achieved when the shift to higher wavelengths appeared. On the other hand-when the laser was coupled with GYAG:Ce ${ }^{3+}$, we observed deterioration of the results. This part of studies showed the importance of keeping the temperature of diode on a constant level and finding an optimum of light conversion by phosphor.

From thermal analysis in FEM software we found out, that operating without cooling system leads to exceeding the junction temperature almost two times. Also, the studies show, that the material of stem in used laser diode could be optimized. We calculated the value of thermal resistance between heat generator and air for conditions with and without cooling module. Investigation proves, that without appropriate cooling module, the value of thermal resistance can increase even three times, which leads to fast diode degradation.

\section{References}

[1] Muthu, S., Schuurmans, F.J. and Pashley, M.D. (2002) Red, Green and Blue Leds for White Light Illumination. IEEE Journal of Selected Topics in Quantum Electronics, 8, 333 https://doi.org/10.1109/2944.999188

[2] Sato, Y., Takahashi, N. and Sato, S. (1996) Full-Color Fluorescent Display Devices Using a Near-uv Light-Emitting Diode. Japanese Journal of Applied Physics, 35, L838-L839. https://doi.org/10.1143/JJAP.35.L838 
[3] Nakamura, S., Pearton, S. and Fasol, G. (2000) The Blue Laser Diode: The Complete Story. SpringerVerlag, Berlin. https://doi.org/10.1007/978-3-662-04156-7

[4] Yang, T.H., Chen, C.-C., Chen, C.-Y., Chang, Y.-Y. and Sun, C.-C. (2014) Essential Factor for Determining Optical Output of Phosphor-Converted LEDs. IEEE Photonics Journal, 6, Article ID: 8200209. https://doi.org/10.1109/JPHOT.2014.2308630

[5] Czesnakowska, A., Ledru, G., Glorieux, B. and Zissis, G. (2017) Effect of Different Phosphor Properties for White Light Generation Using a Blue Laser Diode. Journal of Light \& Visual Environment.

[6] Wen, P.-Y., Li, D.-Y., Znang, S.-M., Liu, J.-P., Zhang, L.-Q., Zhou, K., Feng, M.-X., Tian, A.-Q., Ahang, F., Zeng, C. and Yang, H. (2015) Identification of Degradation Mechanisms Based on Thermal Characteristic of InGaN/GaN Laser Diode. IEEE Journal of Selected Topics in Quantum Electronics, 21, 6.

[7] Meneghini, M., Trivellin, N., Orita, K., Takigawa, S., Tanaka, T., Ueda, D., Meneghesso, G. and Zanoni, E. (2010) Degradation of InGaN-Based Laser Diodes Analyzed by Means of Electrical and Optical Measurements. Applied Physics Letters, $\mathbf{9}$, Article ID: 263501.

[8] Uddin, A., Wei, A.C. and Andersson, T.G. (2005) Study of Degradation Mechanism of Blue Light Emitting Diodes. Thin Solid Films, 483, 378-381. https://doi.org/10.1016/j.tsf.2005.01.018

[9] Retamal, J., et al. (2015) 4-Gbit/s Visible Light Communication Link Based on 16-QAM OFDM Transmission over Remote Phosphor-Film Converted White Light by Using a Blue Laser Diode. Optics Express, 23, 33656. https://doi.org/10.1364/OE.23.033656

[10] Chi, Y.-C., et al. (2015) 450-nm GaN Laser Diode Enables High-Speed Visible Light Comunnication with 9-Gbps QAM-OFDM. Optics Express, 23, 13051. https://doi.org/10.1364/OE.23.013051

[11] Vitaterna, M.H., Takahashi, J.S. and Turek, F.W. (2001) Overview of Circadian Rhythms. Alcohol Research \& Health, 25, 85-93.

[12] Chhajed, S., Xi, Y., Li, Y.-L., Gessman, Th. and Schubert, E.F. (2005) Influence of Junction Temperature on Chromaticity and Color-Rendering Properties of Trichromatic White-Light Source Based on Light-Emitting Diodes. Journal of Applied Physics, 97, Article ID: 054506. https://doi.org/10.1063/1.1852073

[13] Feng, M.-X., Ahang, S.-M., Jiang, D.-S., Liu, J.-P., Wang, H., Zeng, Ch., Li, Z.-Ch., Wang, H.-B., Feng, W. and Yang, H. (2012) Thermal Analysis of GaN Laser Diodes in a Package Structure. Chinese Physics B, 21, 8. https://doi.org/10.1088/1674-1056/21/8/084209

[14] Gen-ichi, et al. (1999) Thermal Analysis of GaN Laser Diodes. Japanese Journal of Applied Physics, 38, 2764-2768.

[15] Osram Opto Semiconductors (2013) Using OSRAM OS Visible InGaN Laser Diode. 